Z. Klin. Chem. Klin. Biochem.

12. Jg. 1974, S. 385-388

\title{
Plasminogengehalt eines im Handel befindlichen Rinderfibrinogens
}

\author{
Von H. Auel und M. Martin
}

Aus der Aggertalklinik. Klinik für Gefäßerkrankungen. (Chefarzt: Prof. Dr. W. Schoop) Engelskirche'n bei Köln

(Eingegangen am 10. Dezember 1973/26. April 1974

Da Rinderfibrinogen die Basis für viele gerinnungsphysiologische Untersuchungen darstellt, wurde mit Hilfe der Fibrin-Plattentechnik und einer Aktivierung des fibrinolytischen Systems durch die direkte Umwandlung des Plisminogens in Plasmin mittels Urokinase untersucht, wieviel Plasminogen dem Rinderfibrinogen anhaftet. Aus dem Verhältnis zwischen Verdunnungsstufen des Rinderfibrinogens und des Humanplasmas mit gleicher fibrinolytischer Aktivitat war zu ersehen, dals die Relation Plasminogen zu Fibrinogen im Rinderfibrinogen (Behring) im Mittel um das 2,7-fache höher lag als in einer Euglobulinfraktion eines norinalen menschlichen Mischplasma $(n=60)$.

\section{The plasminogen cont ent of a commercially available bovine fibrinogen}

Since fibrinogen serves as the basis for many investigations into the physiology of coagulation, its plasminogen cont cnt is of int crest. The plasminogen content was determined with the aid of the fibrin plate technique; the fibrinolytic system was activated by the direct conversion of plasminogen into plasmin with the aid of urokinase. The dilution steps of the bovine fibrinogen and of human plasma with the same fibrinolytic activity were compared. It was shown that the ratio of plasminogen to fibrinogen (Behring) was on average 2.7 times higher than that of a euglobulin fraction from normal human inixed plasma $(n=60)$.

Rinderfibrinogen, die Basis für viele gerinnungsphysiologische Untersuchungen, hat in den Gerinnungslaboratorien einen festen Platz bei der Durchführung der Zweiphasen-Prothrombinbestimmung (1), des Prothrombinverbrauchstests (2), der Plasminogenbestimmung (3) und der quantitativen Streptokinasebestim. mung (4).

Für verschiedene praktische und theoretische Aspekte, insbesondere der fibrinolytischen Testmethoden, war die Frage, wieviel Plasminogen das Rinderfibrinogen der Behringwerke AG, Marburg/Lahn, enthält, von Wichtigkeit.

Im Folgenden wurde versucht, mit Hilfe der FibrinPlattentechnik (5) diese Frage zu beantworten.

Plasminogen ist die inaktive Vorstufe des fibrinolytischen Enzyms Plasmin (EC 3.4.21.7) und kann durch Blutbzw. Gewebsaktivatoren oder Urokinase (EC 3.4.99.26) in dieses umgewandelt werden. Das Substrat von Plasmin stellt u. a. Fibrin dar, welches durch die Enzymwirkung in verschiedene Bruchstücke abgebaut wird.

Mit Hilfe der Fibrinplatten -Methode können derartige fibrinolytische Plasmin-Quantitäten erfaßt werden. Die Aktivierung des fibrinolytischen Systems erfolgte im vorliegenden Fall durch direkte Umwandlung des Plasminogens mit Hilfe von Urokinase. Als Bezugssystem bietet sich die Plasminogenkonzentration von gepooltem Humanplasma an. Hier müssen allerdings vor Umwandlung des Plasminogens in Plasmin die Inhibitoren (Antiplasmine) ausgeschaltet werden. Dies war durch
Ausfällen der Euglobuline nach von Kaulla und McDonald (6) möglich.

\section{Material und Methoden}

Herstellung crhitzter Rinder-Fibrinplatten in Anlehnung an Lassen (7)

\section{Reagenzien}

Rinderfibrinogen, Fläschchen $7.460 \mathrm{mg}$ (Bchringwerkc AG, Marburg/Lahn)

Veronalpuffer pH 7,8 -- Ionenstärke 0,1

Die Stammlösung enthält $0,1 \mathrm{~mol}$ Barbital-Natrium $(20,62 \mathrm{~g})$

und $0,137 \mathrm{~mol} \mathrm{NaCl}$ ad $1000 \mathrm{ml}$ dest. Wasser.

Gebrauchspuffer: $495 \mathrm{ml} \mathrm{Stammlösung,} 255 \mathrm{ml} 0,1 \mathrm{~mol} / \mathrm{l}$ $\mathrm{HCl}$ und $225 \mathrm{ml}$ dest. Wasser

Thrombin: Fläschchen zu 3000 E (Topostasin, Hoffmann-La Roche AG, Grenzach/Baden)

Physiologische $\mathrm{NaCl}$-Lösung

\section{Gang der Herstellung}

$120 \mathrm{mg}$ Rinderfibrinogen wurden in $60 \mathrm{ml}$ Veronalpuffer pH 7,8 gelöst und filtriert (Filter $595, \phi 11 \mathrm{~cm}$, Schleicher \& Schüll, Dassel/Krcis Einbeck).

3000 E Thrombin (Topostasin) wurden in $6,0 \mathrm{ml}$ physiologischer $\mathrm{NaCl}$-Lösung gelöst. $0,5 \mathrm{ml}$ diescr Lösung $+4,5 \mathrm{ml}$ physiologische $\mathrm{NaCl}$-Lösung ergaben $50 \mathrm{E} / \mathrm{ml}$ Thrombin. 7 Petrischalen wurden bereitgestellt. In jede Schale Einpipettieren von $8,0 \mathrm{ml}$ Fibrinogenlösung und unter ständiger Bewegung $0,4 \mathrm{ml}$ Thrombinlösung.

Schalen verschließen, zum Aufnehmen des Kondenswassers Deckel mit Filterpapier auslegen. Dic Fibrinplatten für 1 Stunde bei $37^{\circ} \mathrm{C}$ belassen, anschließend für 45 Minuten bei $85^{\circ} \mathrm{C}$ erhitzen. Abkühlen im Brutschrank, Aufbewahren im Kühlschrank. Die Plasminogenfreiheit wurde stets durch Auftropfen von Urokinaselösung und anschließender Inkubation nachgewiesen. 
Aufarbeitung des Human-Mischplasma, Zubereitung von Euglobulin nach von Kaulla und McDonald (6)

\section{Reagenzien}

Citratplasma von 60 gesunden Probanden (Klinikpersonal, Patienten mit statischen Beschwerden).

9 Teile Blut +1 Teil Natriumcitrat $3,8 \%$, zentrifugiert bei $2.500 \mathrm{U} / \mathrm{min}$ für 10 Minuten.

Michaelis-Puffer pH 7,8 - Ionenstärke 0,1

EDTA-Lösung $10 \mathrm{~g} / 1$

$\mathrm{CO}_{2}$ (Gasflasche)

\section{Zubereitung von Euglobulin}

$1,5 \mathrm{ml}$ Citrat-Mischplasma wurden in einem $50 \mathrm{ml}$ Erlenmeyerkolben mit $22,5 \mathrm{ml}$ dest. Wasser verdünnt. Anschließend 4 Minuten Begasen mit $\mathrm{CO}_{2}$. Der Gasstrom wurde so eingestellt, daß sich der Flüssigkeitsspiegel bewegte, aber nicht schäumte. Bereits nach 1 Minute war eine deutliche Trübung zu erkennen.

Durch die $\mathrm{CO}_{2}$-Begasung war eine sehr konstante Einstellung des pH-Wertes auf 5,32 $\pm 0,12$ zu erzielen. Die Plasmaverdünnung wurde unmittelbar nach dem Begasen für 10 Minuten bei $2.500 \mathrm{U} / \mathrm{min}$ zentrifugiert und der Überstand dekantiert. Anschließend Lösen des Sedimentes in 1,4 ml Michaelis-Puffer $\mathrm{pH} \mathrm{7,8}$ und 0,1 $\mathrm{ml}$ EDTA $(10 \mathrm{~g} / \mathrm{l})$

Herstellen der Rinderfibrinogen-Lösung

8 Fläschchen Rinderfibrinogen unterschiedlicher Chargen (1004 B, 1005 C, 1008 D, 1017 B, 1023 A, 1023 B, 1025 B, $1030 \mathrm{~A}) \mathrm{zu}$ je 60 ìm wurden mit je 5,0 ml Michaelis-Puffer pH 7,8 aufgefüllt und zur Lösung über Nacht im Brutschrank bei $37^{\circ} \mathrm{C}$ belassen.

Das so gelöste Rinderfibrinogen wurde mit Michaelis-Puffer auf $20 \mathrm{ml}$ aufgefüllt und anschließend filtriert (Filter 595, $\phi 11 \mathrm{~cm}$, Schleicher \& Schüll, Dassel/Kreis Einbeck).

\section{Bestimmung des Fibrinogengehaltes}

Der Fibrinogengehalt der einzelnen Rinderfibrinogenlösungen wurde gravimetrisch nach Gram (8) wie folgt ermittelt. 2,0 ml Rinderfibrinogenlösung wurden mit $10 \mathrm{NIH} \mathrm{E}$ Thrombin (gelöst in $0,1 \mathrm{ml}$ physiol. $\mathrm{NaCl}$-Lösung) versetzt und für 2 Stunden im Wasserbad bei $37^{\circ} \mathrm{C}$ belassen. Das Gerinnsel wurde vorsichtig von der Glaswand gelöst, in Filterpapier ausgepreßt und für einige Male in physiol. $\mathrm{NaCl}$-Lösung und anschließend in dest. Wasser ausgewaschen. Nach erneutem Auspressen in Filterpapier wurde das Fibrin nun zweimal in absol. Alkohol und zweimal in Äther zum Entwässern gebracht. Das so gewonnene Fibrin wurde nun für einige Stunden im Brutschrank bei $37^{\circ} \mathrm{C}$ aufbewahrt und dann auf einer Analysenwaage ausgewogen.

Ermittlung der zur Plasminogen-Plasmin-Umwandlung optimalen Urokinasemenge

500.000 CTA-Einheiten Urokinase (Hoffman-La Roche \& Co. Ltd., Basel/Schweiz) wurden in 5,0 ml physiologischer $\mathrm{NaCl}$-Lösung gelöst.

Durch mehrere geometrische Verdünnungsreihen wurden unterschiedliche Urokinase-Konzentrationen erzielt.

$0,1 \mathrm{ml}$ Urokinase-Lösung jeder Verdünnungsstufe wurden mit je $1,0 \mathrm{ml}$ Euglobulinlösung (enthielt Euglobulin von $1,0 \mathrm{ml}$ Mischplasma) versetzt. Anschließend erfolgte die Beimpfung erhitzter Rinder-Fibrinplatten mit 0,05 ml dieser Proben. Nach 18 Stunden Inkubation bei $37^{\circ} \mathrm{C}$ Ermittlung der Urokinase-Konzentration, bei der eine deutliche Abnahme der fibrinolytischen Aktivität zu sehen war.

Bei Urokinase-Konzentrationen von 5.000000 bis $10.000 \mathrm{E} / \mathrm{l}$

Euglobulinlösung wurde immer ein Lysehof mit einem Durchmesser-Quadrat von $118 \mathrm{~mm}^{2}$ registriert. Erst die nachfolgenden Urokinase-Konzentrationen von 6.000 bis $40 \mathrm{E} / 1$ Euglobulinlösung zeigten eine deutliche Abnahme der fibrinolyt ischen Aktivität. Bei einer Urokinase-Konzentration von 40 E/1 Euglobulinlösung lag das Quadrat des Durchmessers des Lysehofs nur noch bei $49 \mathrm{~mm}^{2}$ (Abb. 1). Eine Hemmung der fibrinolytischen Aktivität bei sehr hoher Urokinase-Konzentration (z. B. $1.250000 \mathrm{E} / 1$ Euglobulinlösung) wurde nicht beobachtet.

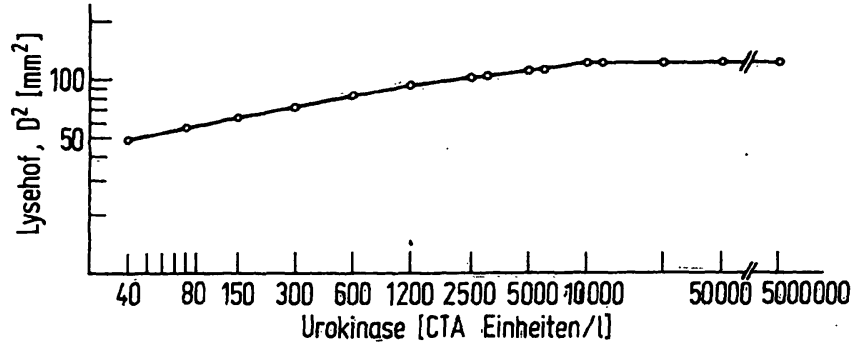

Abb. 1. Ermittlung der zur Plasminogen-Plasmin-Umwandlung optimalen Urokinasemenge mit Hilfe der FibrinplattenMethode. Bei Urokinase-Konzentrationen von $5 \cdot 10^{6}$ bis $1 \cdot 10^{4} \mathrm{E} / 1$ Euglobulinlösung wurde immer eine Lysehof mit einem Durchmesser-Quadrat von $118 \mathrm{~mm}^{2}$ registriert. Erst die nachfolgenden Urokinase-Konżentrationen von $6 \cdot 10^{3}$ bis $40 \mathrm{E} / 1$ Euglobulinlösung zeigten eine deutliche Abnahme der fibrinolytischen Aktivität.

Für die Austestung des Rinderfibrinogens wurde deshalb eine Urokinase-Konzentration von $1.000000 \mathrm{E} / 1$ physiologischer $\mathrm{NaCl}$-Lösung gewählt. Davon wurde $0,1 \mathrm{ml} \mathrm{zu} 1,0 \mathrm{ml}$ Euglobulinlösung bzw. zu 1,0 ml Rinderfibrinogenlösung gegeben.

\section{Ergebnisse}

Fibrinogengehalt der einzelnen Rinderfibrinogenlösungen

Nach gravimetrischer Fibrinogenbestimmung wurden in den einzelnen Rinderfibrinogenlösungen unterschied: licher Chargen folgende Fibrinogen-Konzentrationen ermittelt:

$$
\begin{array}{ll}
\text { Chargen-Nr. } & 1004 \mathrm{~B}-2,95 \mathrm{~g} / 1 \\
& 1005 \mathrm{C}-1,70 \mathrm{~g} / 1 \\
& 1008 \mathrm{D}-2,20 \mathrm{~g} / 1 \\
& 1017 \mathrm{~B}-2,45 \mathrm{~g} / 1 \\
& 1023 \mathrm{~A}-2,55 \mathrm{~g} / 1 \\
& 1023 \mathrm{~B}-2,57 \mathrm{~g} / 1 \\
1025 \mathrm{~B}-2,25 \mathrm{~g} / 1 \\
1030 \mathrm{~A}-2,62 \mathrm{~g} / 1
\end{array}
$$

\section{Plasminogenbestimmung}

\section{Reihe A}

Zur Ermittlung des Plasminogengehaltes von Rinderfibrinnogen wurde zunächst Euglobulinlösung ( $1,0 \mathrm{ml}$ Ëuglobulinlösung enthielt Euglobulin von 1,0 ml Citrat-Mischplasma) im Verhältnis 1:1, 1:2, 1:4 und 1:8 mit Michaelis-Puffer $\mathrm{pH} 7,8$ verdünnt. Je $1,0 \mathrm{ml}$ dieser Verdünnungsstufen wurden mit $100 \mathrm{E}$ Urokinase (in $0,1 \mathrm{ml}$ physiol. NaCl-Lösung) versetzt, davon wurden 0,05 $\mathrm{ml}$ auf die Fibrinplatten gesetzt und nach Inkubation (18 Stunden bei $37^{\circ} \mathrm{C}$ ) die Durchmesser der Lysehöfe gemesssen und deren Quadrat berechnet.

Jede Verdünnungsstufe wurde vierfach ausgetestet, mit Hilfe der Mittelwerte wurde eine Eichgerade auf doppeltlogarithmiertem Papier erstellt (Ordinate: Quadrate der Durchmesser der Lysishöfe; Abszisse: Verdünnungsstufen). 


\section{Reihe B}

Analog zu Reihe A wurden die Rinderfibrinogenlösungen der 8 verschiedenen Chargen ebenfalls im Verhältnis $1: 1,1: 2,1: 4$ und $1: 8$ verdünnt und je $1,0 \mathrm{ml}$ der Verdünnungen mit $100 \mathrm{E}$ Urokinase (in $0,1 \mathrm{ml}$ physiol. $\mathrm{NaCl}$-Lösung) versetzt.

Aufbringen von $0,05 \mathrm{ml}$ des Gemisches auf Fibrinplatten und nach Inkubation ( 18 Stunden bie $37^{\circ} \mathrm{C}$ ) Bestimmung der Lysehofdurchmesser und -Quadrate. Die ermittelten Werte (Einfachbestimmungen) wurden ebenfalls auf doppelt-logarithmiertem Papier aufgetragen und durch Einzeichnen einer Geraden zueinander in Beziehung gebracht.

Es bestanden somit 1 Eichgerade aus vier Verdünnungsstufen einer Euglobulinlösung, sowie je 1 Eichgerade aus vier Verdünnungsstufen der einzelnen Rinderfibrinogenlösungen. Wir beobachteten einen mittleren Wert des Quadrats des Lysehofdurchmessers von $140 \mathrm{~mm}^{2}$. Die Verdünnungen, die eine derartige lytische Aktivität entwickelten, besaßen einen gleichen Plasminogen (bzw. Plasmin) -Gehalt.

Für Reihe A (Referenzwerte aus Human-Euglobulin) war dies eine bestimmte Verdünnung $1:$ a und furr Reihe B (Rinderfibrinogen) $1:$ b. Die Plasminogenkonzentration ( $x \%$ ) in Reihe $B$, ausgedrückt in Konzentration der Reihe A, errechnete sich durch folgenden Ansatz (Abb. 2, Tab. 1):

$$
\frac{100 \%}{\mathrm{a}}=\frac{\mathrm{x} \%}{\mathrm{~b}} \quad \mathrm{x} \%=\frac{100 \mathrm{~b}}{\mathrm{a}}
$$

Um die Plasminogenkonzentrationen im Humanplasma . bzw. in den Rinderfibrinogenlösungen zum Fibrinogengehalt korrelieren zu können, bezogen wir den Plasminogengehalt auf $1,0 \mathrm{~g} / 1$ Fibrinogen.

Für Humanplasma wurde bei 2,84 g/l Fibrinogen (gravimetrisch ermittelt) der Plasminogengehalt als $100 \%$ definiert. Die Plasminogenkonzentration, bezogen auf eine gewählte Fibrinogenkonzentration von $1 \mathrm{~g} / 1$, errechnete sich nach der Formel:

$$
\begin{aligned}
& \frac{100 \%}{2,84 \mathrm{~g} / 1}=\frac{x \%}{1,0 \mathrm{~g} / 1} \\
& x \% \quad \equiv \quad \frac{100}{2,84} \\
& x \%=35,2 \%
\end{aligned}
$$

Für diese Fibrinogenkonzentration $(1,0 \mathrm{~g} / \mathrm{l})$ konnte nunmehr auch der Plasminogengehalt der einzelnen Rinderfibrinogen-Chargen errechnet werden (Tab. 1).

Das Verhältnis von Rinderplasminogen zu Humanplasminogen (bezogen auf $1,0 \mathrm{~g} / 1$ Fibrinogen) wurde bestimmt und in Tabelle 1 zusammengestellt.

Der Mittelwert dieses Verhältnisses betrug $\bar{x} \pm s=2,7$ $\pm 0,87$.

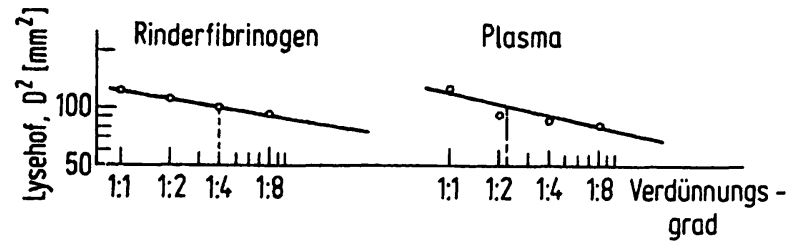

Abb. 2. Beispicl zur Ermittlung des Plasminogengehaltes von Rinderfibrinogen und Human-Mischplasma mit Hilfe der Fibrinplatten-Methode. Die Durchmesser der Lysehöfe wurden gemessen und die Quadrate der Durchmesser

\begin{tabular}{|c|c|c|c|c|}
\hline Charge & $\begin{array}{l}\text { Rinderfibri- } \\
\text { nogen } \\
{[\mathrm{g} / 1]}\end{array}$ & $\begin{array}{l}\text { Plasmino- } \\
\text { gengehalt } x\end{array}$ & $\begin{array}{l}\text { Rinder-Plas- } \\
\text { minogen pro } \\
1 \text { g/l Fibri- } \\
\text { nogen } \\
\\
{[\%]}\end{array}$ & $\begin{array}{l}\text { Verhältnis von } \\
\text { Rinder-Plas- } \\
\text { minogen zu } \\
\text { Human-Plas- } \\
\text { minogen pro } \\
1 \text { g/l Fibri- } \\
\text { nogen }\end{array}$ \\
\hline $\begin{array}{l}1004 \mathrm{~B} \\
1005 \mathrm{C} \\
1008 \mathrm{D} \\
1017 \mathrm{~B} \\
1023 \mathrm{~A} \\
1023 \mathrm{~B} \\
1025 \mathrm{~B} \\
1030 \mathrm{~A}\end{array}$ & $\begin{array}{l}2,95 \\
1,70 \\
2,20 \\
2,45 \\
2,55 \\
2,57 \\
2,25 \\
2,62\end{array}$ & $\begin{array}{l}215 \\
240 \\
210 \\
240 \\
300 \\
200 \\
100 \\
320\end{array}$ & $\begin{array}{r}72,9 \\
141,2 \\
95,5 \\
98,0 \\
117,6 \\
77,8 \\
44,4 \\
122,1\end{array}$ & $\begin{array}{l}2,1: 1 \\
4,0: 1 \\
2,7: 1 \\
2,8: 1 \\
3,3: 1 \\
2,2: 1 \\
1,3: 1 \\
3,5: 1\end{array}$ \\
\hline $\begin{array}{l}\bar{x}= \\
s=\end{array}$ & $\begin{array}{l}2,41 \\
0,37\end{array}$ & & $\begin{array}{r}96,2 \\
\pm \quad 31,0\end{array}$ & $\begin{array}{l}2,7 \\
+0,87\end{array}$ \\
\hline
\end{tabular}
errechnet. Die ermittelten Werte wurden auf doppeltlogarithmiertem Papier aufgetragen und durch Einzeichnen einer Geraden zueinander in Beziehung gebracht.

\section{Besprechung und Zusammenfassung}

Die Frage, wieviel Plasminogen dem Rinderfibrinogen anhaftet, wurde unseres Wissens in der Literatur noch nicht erörtert.

Da Rinderfibrinogen die Basis für viele gerinnungsphysiologische Untersuchungen darstellt, wurde versucht, mit Hilfe der Fibrin-Plattentechnik und einer Aktivierung des fibrinolytischen Systems durch die direkte Umwandlung des Plasminogens in Plasmin mittels Urokinase diese Frage zu beantworten.

Nach Ermittlung des zur Plasminogen-Plasmin-Umwandlung optimalen Urokinasegehaltes erfolgte die Gegenüberstellung einer konstanten Menge Rinderfibrinogen mit einem Human-Mischplasma in Hinsicht auf deren Plasminogengehalt.

Die ermittelten Quadrate der Durchmesser der Lysehöfe wurden auf doppeltlogarithmiertem Papier aufgetragen und durch Einzeichnen einer Geraden zueinander in Beziehung gebracht.

Aus dem Verhältnis zwischen Verdünnungsstufen des Rinderfibrinogens und des Humanplasmas mit gleicher fibrinolytischer Aktivität war zu ersehen, daß die Relation Plasminogen zu Fibrinogen im Rinderfibrinogen (Behring) im Mittel um das 2,7-fache höher lag als in einer Euglobulinfraktion aus normalem menschlichem Mischplasma $(n=60)$. 


\section{Literatur}

1. Schultze, H. E. \& Schwick, G. (1953), Medizinische Nr. 42 u. 43 , S. 1354 u. 1386.

2. Quick, A. J. (1947), Amer. J. Med. Sci. 214, 272-280.

3. Martin, M. (1969), Thromb. Diath. Haemorrh. 22, 121-137.

4. Martin, M., 80. Verhandl. d. Dtsch. Ges. inn. Med. 21-25. April 1974, Wiesbaden.

5. Astrup, T. \& Müllertz, S. (1952), Arch. Biochem. 40, $346-351$.

6. v. Kaulla, K. N. \& Mc Donald, T. S. (1958), Blood 13, $811-821$.

7. Lassen, M. (1952), Acta Physiol. Scand. 27, 371-376.

8. Gram, H. C. (1921), J. Biol. Chem. 49, 279-295.

H: Auel und Priv.-Doz. Dr. M. Martin 5250 Engelskirchen Aggertalklinik 